\title{
Comparative study of sFlt-1/Plgf ratio with uterine artery doppler indices in prediction of preeclampsia at 22-24 weeks period of gestation
}

\author{
Taru Gupta $^{1}$, Pooja Sharma ${ }^{1 *}$, Sarika Arora ${ }^{2}$, Divya Baruhee ${ }^{1}$
}

\begin{abstract}
${ }^{1}$ Department of Obstetrics and Gynecology, ESI-PGIMSR Basaidarapur, New Delhi, India
${ }^{2}$ Department of Biochemistry, ESI-PGIMSR Basaidarapur, New Delhi, India
\end{abstract}

Received: 05 January 2020

Accepted: 15 January 2020

\author{
*Correspondence: \\ Dr. Pooja Sharma, \\ E-mail: poojasharma20091990@gmail.com
}

Copyright: (c) the author(s), publisher and licensee Medip Academy. This is an open-access article distributed under the terms of the Creative Commons Attribution Non-Commercial License, which permits unrestricted non-commercial use, distribution, and reproduction in any medium, provided the original work is properly cited.

\begin{abstract}
Background: Hypertensive disorders like pre-eclampsia along with hemorrhage and infection, contributes greatly to maternal morbidity and mortality. Various pro and antiangiogenic factors like sFlt-1 and Plgf have been linked to the etiopathogenesis of placental vascular disease and their combination with uterine artery doppler studies may improve the prediction accuracy. Present study was conducted to analyze sFlt-1/Plgf ratio and uterine artery doppler indices among high risk patients and to compare these in prediction of preeclampsia.

Methods: A prospective observational study was conducted from September 2017 to February 2019 in which 100 patients giving consent and satisfying inclusion criteria were evaluated for various risk factors and were subjected to sFlt-1/Plgf ratio test and uterine artery doppler study at 22-24 weeks period of gestation. They were followed up and maternal outcome was analysed.

Results: Among the cohort of 100 women with high risk factors, 35\% of the study participants developed preeclampsia. Using sFlt-1/Plgf ratio $40 \%$ of them were screened positive for pre-eclampsia. This percentage of screened positive was $40 \%, 43 \%$, and $53 \%$ using uterine artery RI, PI, and SD respectively. sFlt-1/Plgf was found to have a sensitivity of $91.4 \%$ and specificity of $87.7 \%$. ROC curve analysis showed highest area under curve (AUC) for sFlt1/Plgf (0.858).

Conclusions: sFlt-1/Plgf ratio was found to be a better predictable biomarker than uterine artery Doppler indices in prediction of pre-eclampsia at 22-24 weeks period of gestation.
\end{abstract}

Keywords: High risk, Pre-eclampsia, Resistive index, Systolic to diastolic, sFlt-1/Plgf, Uterine artery

\section{INTRODUCTION}

Hypertensive disorders complicate 5 to 10 percent of all pregnancies, and together they are one of the deadly triad along with hemorrhage and infection, contributing greatly to maternal morbidity and mortality. Preeclampsia either alone or superimposed on chronic hypertension, is the most dangerous. ${ }^{1}$

A variety of pro and antiangiogenic factors involved in placental vascular development have been studied. The families of vascular endothelial growth factor (VEGF) and angiopoietin (Ang) are most extensively studied among other factors.

Soluble Fms-like tyrosine kinase 1 (sFlt-1) is a variant of the Flt-1 receptor for placental growth factor (Plgf) and for VEGF. Enhanced placental expression and secretion of sFlt-1 appear to play a major role in the development of preeclampsia. ${ }^{2}$ Soluble fms-like tyrosine kinase-1 (sFlt-1) binds vascular endothelial growth factor- A (VEGF-A) and placental growth factor (Plgf) which are the main angiogenic factors, responsible for placental vascular development and maternal endothelial function, and in this way prevents their interaction with 
endogenous receptors in the vessels. ${ }^{3}$ Because of that, circulating Plgf levels are decreased and sFlt-1 levels are elevated. ${ }^{4}$ Ratio of these two factors has proven to be more accurate for prediction of preeclampsia than any of these factors alone. . $^{5-8}$

Combining Plgf concentrations with other biochemical markers, uterine artery doppler studies, or both, substantially improves the predictive value. ${ }^{9}$

Uterine artery doppler waveform analysis also has the best predictability at 22-26 weeks period of gestation. ${ }^{10}$ Resistance to blood flow within uteroplacental circulation is transmitted upstream to the uterine arteries. ${ }^{11}$ Faulty trophoblastic invasion of the spiral arteries results in diminished placental perfusion and upstream increased uterine artery resistance; resulting in increased uterine artery pulsatility index (PI) or resistance index (RI) and persistence of early diastolic notch. ${ }^{1}$

As limited studies have been done for prediction of preeclampsia by sFlt-1/Plgf ratio. We planned to do comparative study between sFlt-1/Plgf ratio and uterine artery doppler indices for prediction of preeclampsia at 22-24 weeks period of gestation.

\section{METHODS}

A prospective observational study was conducted from September 2017 to February 2019 at the department of obstetrics and gynaecology and department of biochemistry ESIC-PGIMSR Basaidarapur, New Delhi. Antenatal patients at 22-24 weeks period of gestation with essential hypertension, overt diabetes, renal disease, autoimmune diseases, history of abruption, fetal growth restriction, unexplained IUFD, preterm delivery, elderly primigravida and bad obstetrics history were included in the study. Patients who had gestational diabetes mellitus, prelabour rupture of membranes, molar pregnancy, multifetal pregnancy and those who did not consent were excluded from the study.

The study protocol was approved from Institutional ethical Committee, ESIC-PGIMSR Basaidarapur, New Delhi. All patients were informed about the study procedure and confidentiality regarding data. Written consent was taken from each participant. Each patient had a right to withdraw at any stage from the study.

\section{Sample size calculation}

Previous studies conducted in the similar settings to ours for prediction of pre-eclampsia had the sensitivity of serum biomarkers combined with uterine artery doppler indices ranging from $70 \%$ to $90 \%$. Considering the observational nature of present study, sample size was calculated using formula:

$\mathrm{N} \geq \mathrm{Zpq} / 12$
Assuming $\mathrm{p}=85 \%$ and taking $7 \%$ margin of error, applying the above formula give sample size to be 100 patients.

\section{sFlt-1/Plgf ratio estimation}

Maternal serum levels of sFlt-1 and Plgf (both levels measured in picograms per milliliter) were determined by means of the fully automated Elecsys assays on an electrochemiluminescence immunoassay platform (cobas e analyzers Roche diagnostics) and were used to calculate the sFlt-1: Plgf ratio.

\section{Uterine artery doppler ultrasonography}

At 22-24 weeks period of gestation, a transabdominal doppler ultrasound (with $3.5 \mathrm{MHz}$ curvilinear probe) of uterine artery velocity waveforms were performed on a woman using an ultrasound machine (Toshiba). Pulsed wave doppler was then used with a sampling gate set of 2 $\mathrm{mm}$ to cover the whole uterine artery of both sides. When three similar consecutive waveforms were obtained, the uterine artery PI (Pulsatality index), RI (Resistance index), S/D ratio was calculated, and the mean doppler values of the uterine arteries were calculated.

All the females were followed up at 2 weekly intervals to detect preeclampsia or any other adverse maternal outcome at the earliest. The early onset and late onset preeclampsia were recorded and managed as per local institutional protocol.

\section{Statistical analysis}

All the data was entered in MS-Excel and statistical analysis was done on SPSS 17.0. Sensitivity analysis was done for various screening tools and ROC curves were plotted for each of them.

\section{RESULTS}

At enrolment detailed history of study participants were asked about various clinical history variables and their responses were recorded. Following observations were seen. Study participants were assessed for various risk factors (essential hypertension, overt diabetes, renal disease, autoimmune diseases) based on history (abruption, fetal growth restriction, unexplained IUFD, preterm delivery, elderly primigravida and bad obstetrics history). Unexplained intra-uterine fetal death was found to be most common (24\%) risk factor among all. This was followed by bad obstetric history (14\%) (Figure 1).

Out of 100 participants enrolled in the study, 35 patients (35\%) developed preeclampsia (Table 1). Majority $(51.4 \%)$ of study participants had late onset of preeclampsia and about $48.6 \%$ of them had early onset of pre-eclampsia. $23 \%$ of participants developed non-severe preeclampsia and $12 \%$ developed severe preeclampsia. 
Table 1: Distribution of study participants according to blood pressure, urine analysis, blood sugar status, liver enzyme values at the time of diagnosis of preeclampsia $(\mathrm{N}=\mathbf{1 0 0})$.

\begin{tabular}{|lll|}
\hline Parameters & Frequency &.$\%$ \\
\hline Normotensive & 65 & 65 \\
\hline Non severe preeclampsia & 23 & 23 \\
\hline Severe preeclampsia & 12 & 12 \\
\hline Urine albumin 1+ & 25 & 25 \\
\hline Urine albumin 2+ & 10 & 10 \\
\hline Deranged GTT $(140-200 \mathrm{mg} / \mathrm{dl})$ & 02 & 2 \\
\hline $\begin{array}{l}\text { Deranged LFT }(\text { SGOT }>\text { 50, } \\
\text { SGPT }>\text { 50) }\end{array}$ & 20 & 20 \\
\hline
\end{tabular}

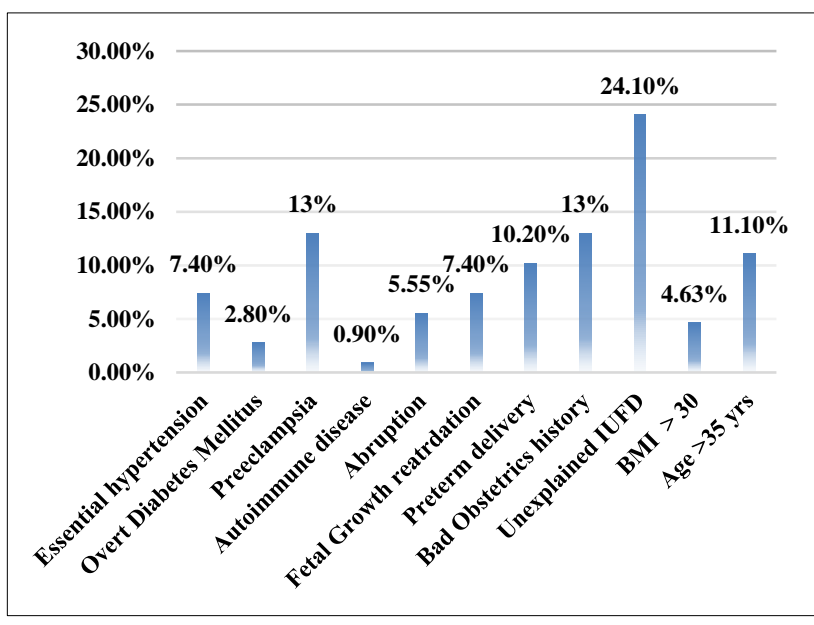

Figure 1: Risk factors observed, pre-disposing study participants to pre-eclampsia.

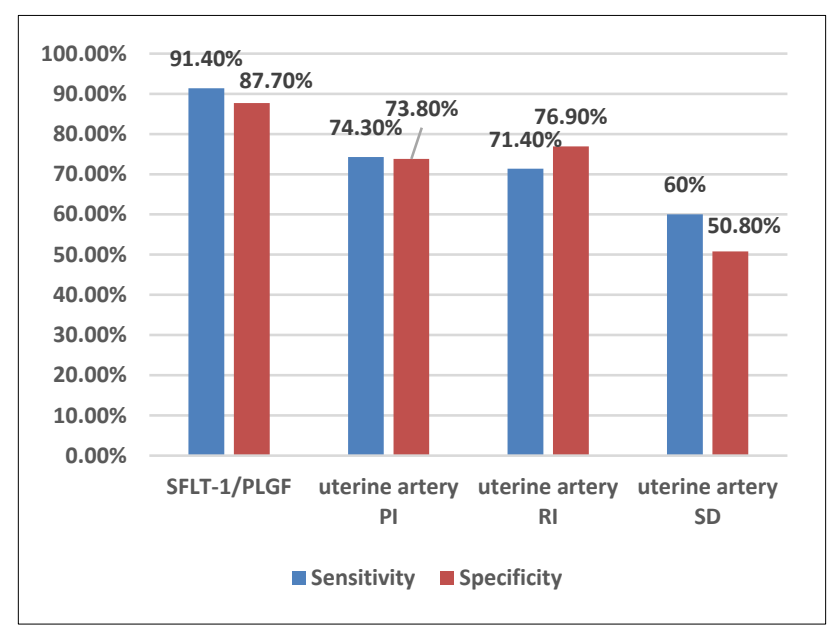

Figure 2: Comparing sensitivity and specificity of various predictor tools.

All study participants were tested with sFlt-1/Plgf ratio and uterine artery doppler study at 22-24 weeks period of gestation. It was observed that 40 participants were screened positive for pre-eclampsia by sFlt-1/Plgf ratio (cut off $>25$ ) and among them 32 developed preeclampsia. This number of screened positive was similar in participants screened positive by resistance index (40) (cut off > 0.7) and 25 among them developed preeclampsia. Using PI 43 participants were screened positive for pre-eclampsia (cut off $>1.1$ ) and among them 26 participants developed preeclampsia as compared to 53 participants who were screened positive (cut off $>2.6$ ) when SD (systolic to diastolic) ratio among them only 21 patients developed preeclampsia.

sFlt-1/Plgf was found to be a good predictor tool with a sensitivity of $91.4 \%$ and specificity of $87.7 \%$. Uterine artery RI, PI and SD had their sensitivities as $71.4 \%$, $74.3 \%$ and $60.5 \%$ respectively, while their specificities were $76.9 \%, 73.8 \%$ and $50.8 \%$ respectively (Figure 2).

It was observed that sFlt-1/Plgf had highest positive predictive value (PPV) of $80 \%$ as well as best negative predictive value (NPV) of $95 \%$ amongst all.

ROC curve analysis depicted highest area under curve (AUC) for sFlt-1/Plgf [0.858 (95\% CI: 0.777-0.939) (p value - 0.041)]. This was found to be significantly good in prediction of preeclampsia. AUC for PI was found to be significantly good [0.697 (95\% CI: 0.581-0.809) (p value - 0.001)]. For RI and SD AUC values were poor [0.695 (95\% CI: 0.586-0.608)] and failed [0.551 (95\% CI: 0.435-0.666)] respectively (Figure 3).

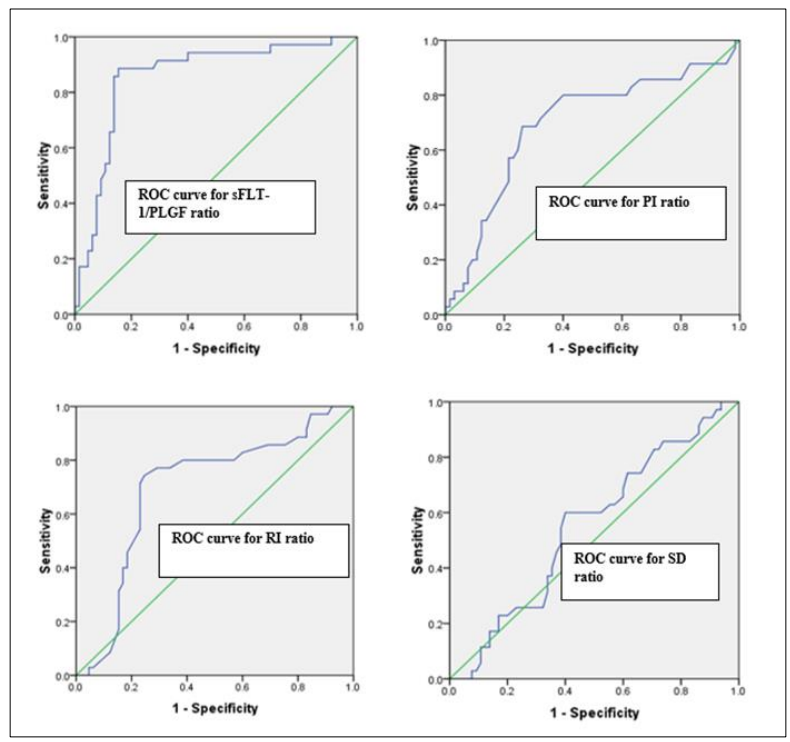

Figure 3: ROC Curve analysis of sFlt-1/Plgf ratio and uterine artery doppler indices.

\section{DISCUSSION}

It was observed that $35 \%$ of the study participants enrolled in the study developed pre-eclampsia. Lijie Li et al, studied sFlt-1/Plgf ratio for prediction of preeclampsia and found the incidence of preeclampsia to be $5.1 \%$, high incidence of preeclampsia in our study was because the study population enrolled was a cohort with risk factors or with history of maternal adverse event. ${ }^{11}$ Perales A et 
al, in their study using the same ratio found that $19.64 \%$ developed preeclampsia. ${ }^{12}$ They also reported that $35.5 \%$ study participants with history of preeclampsia in previous pregnancy developed early onset preeclampsia. These results differ from that of the current study, where $48.6 \%$ had early onset preeclampsia.

In the study $2 \%$ of the women had deranged blood sugar level. Vellamkondu A et al, conducted a study on risk assessment at 11-14 weeks gestation and observed that $8.63 \%$ of patient had deranged blood sugar levels. ${ }^{10}$ Present study has reported $20 \%$ of the participants with raised liver enzyme values (SGOT > 50, U/L SGPT > 50 U/L). Elbishry GM et al, conducted a study in which they reported that $22 \%$ of the patients with preeclampsia had deranged LFT. ${ }^{13}$

In the current study, 40 patients were screened positive by sFlt-1/Plgf ratio and by RI and amongst them 32 patients and 25 patients were diagnosed as preeclampsia respectively. 43 patients were screened positive with uterine artery PI and of them 26 developed preeclampsia. 53 patients were screened positive with uterine artery SD and among them 21 patients developed preeclampsia.

In our study sensitivity and specificity of sFlt-1/Plgf ratio was found to be $91.4 \%$ and $87.7 \%$ respectively. The sensitivity and specificity of PI is $74.3 \%$ and $73.8 \%$ respectively. While in a similar study conducted by Perales A et al, found that sFlt-1/Plgf ratio was better predictor for preeclampsia than uterine artery doppler pulsatility index with sensitivity and specificity of $91.1 \%$ and $82 \%$ respectively similar to our study. ${ }^{12}$ Taraseviciene $\mathrm{V}$ et al in the study on prediction of preeclampsia found that sensitivity and specificity of sFlt-1/Plgf was $95.8 \%$ and $96.2 \%$ respectively. ${ }^{14}$ Gomez A et al did a study using sFlt-1/Plgf ratio and found a sensitivity of $64 \%$ and $95 \%$ specificity to predict preeclampsia. $^{15}$

Narang $\mathrm{S}$ et al, conducted a study on prediction of preeclampsia at 11-14 weeks of pregnancy using uterine artery doppler indices found similar sensitivity (75.9\%) and specificity $(79.6 \%)$ of uterine artery doppler PI as reported in the present study. ${ }^{16}$

sFlt-1/Plgf had highest positive predictive value (PPV) of $80 \%$ as well as best negative predictive value (NPV) of $95 \%$. Zeisler $\mathrm{H}$ et al did a study in which they found that NPV of sFlt-1/Plgf was $99.3 \%$ which is almost like our study. ${ }^{17}$ In our study positive predictive value for PI was $60.46 \%$ and negative predictive value was $84.21 \%$.

\section{CONCLUSION}

sFlt-1/Plgf ratio was found to be a better predictable biomarker than uterine artery doppler indices in prediction of pre-eclampsia at 22-24 weeks period of gestation. Hence sFlt-1/Plgf ratio may be a promising tool for prediction of preeclampsia and thus may aid in the management to improve maternal and fetal outcome.

Funding: No funding sources

Conflict of interest: None declared

Ethical approval: The study was approved by the Institutional Ethics Committee

\section{REFERENCES}

1. Kenneth J Leveno (ed.). Williams Manual of Pregnancy Complications. Hypertensive disorders. $23^{\text {rd }}$ Edition. New York: McGraw Hill Professional; 2013:728.

2. Staff AC, Johnsen GM, Dechend R, Redman CW. Preeclampsia and uteroplacental acute atherosis: immune and inflammatory factors. J Reprod Immunol. 2014;101-102:120-6.

3. Brownfoot FC, Hastie R, Hannan NJ, Cannon P, Tuohey L, Parry LJ, et al. Metformin as a prevention and treatment for preeclampsia: effects on soluble fms-like tyrosine kinase 1 and soluble edoglin secretion and endothelial function. Am J Obstet Gynecol. 2016;214(3):356.e1-356.e15.

4. Lecarpentier E, Vieillefosse S, Haddad B, Fournier $\mathrm{T}$, Leguy MC, Guibourdenche J, et al. Placental growth factor (PIGF) and s-Flt-1 during pregnancy: physiology, assay and interest in preeclampsia. Ann Biol Clin (Paris). 2016;74(3):259-67.

5. Herraiz I, Llurba E, Verlohren S, Galindo A. Update on the diagnosis and prognosis of preeclampsia with the aid of the sFlt-1/ PlGF ratio in singleton pregnancies. Fetal Diagn Ther. 2018;43:81-9.

6. Park HJ, Kim SH, Jung YW, Shim SS, Kim JY, Cho YK, et al. Screening models using multiple markers for early detection of late-onset preeclampsia in lowrisk pregnancy. BMC Preg Childbirth. 2014;14(1):35.

7. Van Helden J, Weiskerchen R. Analytical evolution of the novel soluble fms-like tyrosine kinase- 1 and placental growth factor assays for diagnosis of preeclampsia. Clin Biochem. 2015;48(16-17):11139.

8. Zeisler H, Llurba E, Chantraine F, Vatish M, Staff AC, Sennström M, et al. Predictive value of the sFlt1:PlGF ratio in women with suspected preeclampsia. New Eng J Med. 2016;374(1):13-22.

9. Velauthar L, Plana MN, Kalidindi M, Zamora J, Thilaganathan B, Illanes SE, et al. First-trimester uterine artery Doppler and adverse pregnancy outcome: a meta-analysis involving 55974 women. Ultrasound Obstet Gynecol, 2014;43:500-7.

10. Vellamkondu A, Vasudeva A, Bhat RG, Kamath A, Amin SV, Rai L, et al. Risk assessment at 11-14week antenatal visit: a tertiary referral center experience from south India. J Obstet Gynaecol India. 2017;67(6):421-7.

11. Li L, Zheng Y, Zhu Y, Li J. Serum biomarkers combined with uterine artery Doppler in prediction 
of preeclampsia. Exp Ther Med. 2016;12(4):251520.

12. Perales A, Delgado JL, de la Calle M, GarcíaHernández JA, Escudero AI, Campillos JM, et al. sFlt-1/PLGF for prediction of early-onset preeclampsia: STEPS (Study of Early Pre-eclampsia in Spain). Ultrasound Obstet Gynecol. 2017;50(3):37382.

13. Elbishry GM, Serag Eldin IF, ElShahawy AA, Hawwary GE, Riad AM. Role of soluble FMS-like tyrosine kinase (SFLT-1) /placental growth factor (Plgf) ratio as prognostic marker for cases of preeclampsia. J Gynecol Res Obstet. 2017;3(2):03745.

14. Tarasevičienè V, Grybauskienė R, Mačiulevičienė R. sFlt-1, PlGF, sFlt-1/PlGF ratio and uterine artery Doppler for preeclampsia diagnostics. Medicina. 2016:52(6):349-53.

15. Gomez-Arriaga PI, Herraiz I, Lopez-Jimenez EA, Escribano D, Denk B, Galindo A. Uterine artery Doppler and sFlt-1/PIGF ratio: prognostic value in early-onset pre-eclampsia. Ultrasound Obstet Gynecol. 2014;43:525-32.

16. Narang S, Agarwal A, Das V, Pandey A, Agrawal S, Ali W. Prediction of preeclampsia at 11-14 weeks of pregnancy using mean arterial pressure, uterine artery Doppler and pregnancy associated plasma protein-A. Int J Reprod Contracept Obstet Gynecol. 2016;5(11):3948-53.

17. Zeisler H, Llurba E, Chantraine F, Vatish M, Staff AC, Sennström M, et al. Predictive value of the sFlt1:PIGF ratio in women with suspected preeclampsia. New Eng J Med. 2016;374(1):13-22.

Cite this article as: Gupta T, Sharma P, Arora S, Baruhee D. Comparative study of sFlt-1/Plgf ratio with uterine artery doppler indices in prediction of preeclampsia at 22-24 weeks period of gestation. Int J Reprod Contracept Obstet Gynecol 2020;9:906-10. 